\title{
LA BUENA GOBERNANZA DE LAS FINANZAS PÚBLICAS Y EL DESARROLLO ECONÓMICO
}

\author{
GOOD GOVERNANCE OF PUBLIC FINANCE AND \\ ECONOMIC DEVELOPMENT
}

\author{
Manuel Tirard* \\ Université Paris-Nanterre, Francia
}

\section{Resumen}

El concepto de buen gobierno de las finanzas públicas surgió en los años noventa dentro del discurso de las organizaciones internacionales para promover temas bien conocidos hoy, como el rendimiento del gasto público, la transparencia del presupuesto público, etc. Tras la crisis del 2008, este concepto ha evolucionado hacia la obligación de respetar el equilibrio presupuestario. En todo caso, se debe contrastar la recepción nacional de las prescripciones internacionales en materia de buen gobierno de las finanzas públicas, lo que mostrará las dificultades que implica querer aplicar las mismas reglas jurídicas en todo lugar. El ejemplo de la recepción diversa de la regla de oro de las finanzas públicas por las legislaciones de los Estados europeos lo confirma.

Palabras clave: buen gobierno, finanzas públicas, equilibrio presupuestario.

\section{Abstract}

The concept of good governance of public finances emerged in the 1990s within the discourse of international organizations to promote issues well known today, such as the performance of public spending, the transparency of the public budget, etc. After the 2008 crisis, this concept has evolved * Traducción del original en francés: Doctor Carlos González Palacios (Universidad ESAN,
Perú). 
towards the obligation to respect the budget balance. In any case, the national reception of the international prescriptions in the matter of good governance of public finances must be verified, thus showing the difficulties involved in wanting to apply the same legal rules everywhere. The example of the varied reception of the golden rule of public finances by the laws of European states confirms this.

Keywords: Good governance, public finances, budget balance.

\section{Introducción}

Si bien las empresas privadas se encuentran en el corazón de la actividad y del desarrollo económicos, la intervención pública es también esencial en, al menos, dos aspectos. En primer lugar, hay ocasiones en las que el Estado tiene que constituirse en sí mismo como actor económico en ciertos sectores (grandes obras, energía, etc.), pero también - y sobre todo- debe catalizar el crecimiento, instaurando un marco jurídico favorable para las empresas y para las iniciativas privadas.

Fue dentro de este marco que, desde los últimos años de la década de los ochenta, se utilizó, primero para los países en vías de desarrollo (PVD), el concepto gobernanza, entendida esta como buena por definición por el Banco Mundial ${ }^{1}$. Se trataba de romper con el modelo histórico del gobierno intervencionista -implícitamente entendido como omnipresente e, incluso, autoritario- y sugerir que, en adelante, ya no debía imponer, sino más bien interactuar con el mundo de las empresas y, más ampliamente, con la sociedad civil y los ciudadanos. Es así como en los años noventa, las instituciones internacionales (primero el Banco Mundial y luego el Fondo Monetario Internacional) insistieron en el rol de las instituciones públicas en el desarrollo, sobre todo económico, y en la necesidad de contar con un Gobierno e instituciones públicas estables y eficaces para generar este desarrollo, pensado, primero y antes que nada, desde el sector privado.

Como continuidad a esto, se impuso la idea de una buena gobernanza aplicada a las finanzas públicas o, dicho en otras palabras, la idea de que los

1 La publicación de referencia es: The World Bank, Sub-Saharan Africa: From crisis to sustainable growth, a long-term perspective study (Washington DC: The World Bank, 1989), 60. 
Estados - y no solo los PVD — debían reformar sus presupuestos y aplicar estándares comunes, esencialmente enfocados en el desempeño y la eficacia, pensados para permitir un crecimiento económico perenne, así como para que los inversionistas pudieran encontrar prácticas similares en todos lados. Sin embargo, después del 2008 y de la crisis económica y financiera mundial, este tema ha evolucionado, para expresarlo sintéticamente, hacia una lucha contra los déficits presupuestarios y las deudas públicas, principalmente en los países desarrollados.

El concepto general de «buena gobernanza» aplicado a las finanzas públicas ha sufrido transformaciones muy reales desde esa fecha. Para comprenderlo y entender lo que es vinculante en materia financiera, se necesita revisar primero las evoluciones de este concepto desarrollado por las instituciones internacionales desde hace algunas décadas (2), para luego evaluar la recepción de dichas recomendaciones por los distintos Estados. Sin embargo, si bien es cierto que las prescripciones son las mismas en todos lados, sus efectos deben ser contrastados en cada una de las legislaciones nacionales (3).

\section{El concepto de «buena gobernanza de las finanzas públicas» y sus evoluciones}

Este concepto y su temática, iniciados en los años noventa por las organizaciones internacionales de desarrollo (2.1), fueron renovados frente a la crisis de las finanzas públicas en los últimos diez años (2.2).

\subsection{La aparición y el desarrollo del concepto (décadas de 1990 y 2000)}

Distintas organizaciones internacionales se interesaron por la buena gobernanza, en general, y por aquella concerniente a las finanzas públicas, en particular, defendiendo la idea de que se debían adoptar estándares en los PVD para favorecer el desarrollo - sobre todo el económico-y dar a los ciudadanos confianza respecto de la acción pública. Fue así como el FMI², el Banco

2 Fonds Monétaire International (FMI), Code de bonnes pratiques en matière de transparence des finances publiques (FMI, 1998) (actualizado en el 2007). 
Mundial $^{3}$ o la Organización para la Cooperación y el Desarrollo Económicos ${ }^{4}$ (OCDE) adoptaron diversos documentos, muchas veces múltiples, que insistían en establecer y respetar «buenas» reglas financieras.

Cualesquiera que sean las instituciones y las diferencias en sus enfoques, lo que llama la atención son más bien los puntos en común. Para sintetizar, podríamos decir que todas defienden políticas financieras consideradas como sanas y representativas de una buena gobernanza en la materia. Para resumirlas, se pueden citar los tres aspectos conexos siguientes:

- Primero que nada, transparencia en los datos (impuestos, gastos, contabilidad pública) y en las reglas presupuestarias, entre los tres poderes del Estado (legislativo, ejecutivo y judicial), lo que permite una mejor información, tanto para los inversionistas como para los ciudadanos.

- Luego, controles y procedimientos presupuestarios que sirvan de garantía para el cumplimiento del primer aspecto. Se señala en particular la importancia de un control y de una auditoría externos que permitan al Parlamento acceder a información sobre las prácticas presupuestarias de un poder ejecutivo que tradicionalmente domina la elaboración y la ejecución de los presupuestos públicos. Se trata aquí de garantizar la responsabilidad de los gobernantes (la accountability estadounidense).

- Por último - y globalmente también-, está la necesidad de demostrar cierto «desempeño» en las finanzas públicas, es decir, que el dinero recaudado mediante tributos y luego gastado ha seguido objetivos precisos determinados a priori y alcanzado resultados controlados a posteriori.

Se consideró que estas prescripciones, elaboradas en las décadas de 1990 y 2000, debían ser aplicadas por todos los países. Durante la última década, sin embargo, ante la crisis mundial de las finanzas públicas, sufrieron una transformación que, si bien no hizo desaparecer las recomendaciones iniciales, las completó, renovando, de esta manera, el núcleo del concepto estudiado.

3 Las referencias aquí son múltiples.

4 Diferentes documentos han sido elaborados a partir de 1997 por la OCDE: en materia de tercerización de los servicios públicos, de evaluación de los servicios públicos o incluso y, sobre todo, de transparencia presupuestaria, como «Transparence budgétaire: Les meilleures pratiques de l'OCDE», Revue de l'OCDE sur la gestion budgétaire $1, \mathrm{n}^{\circ} 3(2001 / 2002)$. 


\subsection{La renovación del concepto frente a la crisis de las finanzas públicas del 2008}

La temática de la «buena gobernanza de las finanzas públicas» ha evolucionado desde el año 2008 y a partir de la crisis económica y financiera. Los cambios se efectuaron sobre todo pensando en los países desarrollados, y esto va más allá de la doctrina de las organizaciones internacionales citadas anteriormente.

Esta crisis generó un aumento en los niveles de déficit y deuda públicos en la mayor parte de países ${ }^{5}$. Si se toma el ejemplo de Francia (ya que a Alemania le fue mucho mejor, cabe resaltarlo), nos encontraremos ante un país que practica de forma continua el déficit público — más gastos que ingresos- desde hace aproximadamente 40 años. Este, sin embargo, se incrementó mucho después de la crisis del 2008, y hoy en día alcanza, aproximadamente, el 3\% del PBI francés, porcentaje que se puede explicitar precisando que asciende a 70,000 millones de euros por año. En otras palabras, todas las colectividades públicas de Francia (Estado, colectividades locales y organismos de seguridad social) gastan todavía esa cifra por encima de todos los ingresos que perciben.

Evidentemente, este déficit debe ser financiado, lo que supone recurrir al préstamo, mecanismo que está vigente en Francia desde hace más de 40 años consecutivos. Para darse cuenta de la magnitud de la situación basta precisar que la deuda francesa acumulada supera los 2.3 billones de euros, es decir, el $100 \%$ del PBI francés, una deuda que se ha duplicado en diez años.

Los préstamos, sin embargo, no dejan de tener consecuencias. Por un lado, incrementan continuamente una deuda que, en sí, genera intereses crecientes. Por otro - y esto es importante sobre todo para el tema que se está tratando-, la lucha contra los déficits necesita un importante volumen de impuestos que recargan la economía, especialmente cuando están relacionados con las empresas; pero no solamente los tributos que pagan estas, sino también las aportaciones sociales para financiar la salud, la jubilación, etc. Tales aportaciones son importantes en Francia, lo cual lleva a una competitividad relativa por parte de las empresas francesas.

5 Ver, por ejemplo, https://data.oecd.org/fr/gga/dette-des-administrations-publiques. htm\#indicator-chart 
Para muchos, existen en estas prácticas presupuestarias crónicamente deficitarias la huella de una mala gestión pública, así como también peligros para el desarrollo económico, lo cual necesita reformas. Fue así como el discurso inicial sobre la buena gobernanza de las finanzas públicas se vio completado por una necesidad de limitar el gasto público, o al menos de equilibrarlo con ingresos que, en un entorno globalizado, son cada vez más restringidos y difíciles de incrementar. A esta voluntad de equilibrar el presupuesto se la denomina «la regla de oro de las finanzas públicas».

La buena gobernanza de las finanzas públicas se piensa de esta forma desde hace una década. Se considera que, en general, debe producir en los Estados «buenas prácticas financieras», tal como se sintetiza más arriba; también - y cada vez más - se piensa que se debe generar una lucha contra los déficits presupuestarios y la deuda pública, sobre todo en los países desarrollados. Esta insistencia es fuerte en la Unión Europea, principalmente en lo que respecta al Tratado de Estabilidad, Coordinación y Gobernanza en la Unión Económica y Monetaria (TSCG), firmado en el 2012.

Como puede verse, la crisis de las finanzas públicas de los últimos diez años transformó la idea de buena gobernanza de las finanzas públicas centrándola, más allá de los PVD, en una lógica de supervisión de las finanzas públicas nacionales. Dicho esto, quedan aún por evaluar los efectos concretos. Sin embargo, en este punto, hay que relativizar particularmente la idea de uniformidad.

\section{Un concepto de efectos contrastados en las legislaciones nacionales}

La recepción nacional de las prescripciones internacionales en cuanto a buena gobernanza de las finanzas públicas debe ser contrastada, lo que mostrará las dificultades que implica querer aplicar las mismas reglas jurídicas en todos lados (3.1). El ejemplo de la recepción variada de la regla de oro de las finanzas públicas por las legislaciones de los Estados europeos lo confirmará (3.2).

\subsection{La dificultad de aplicar estándares internacionales en el ámbito nacional}

Aquí no se trata de abordar todos los países, sino más bien de mostrar los dilemas generales que reflejan que las finanzas públicas se inscriben en tradiciones 
(sobre todo jurídicas) y en realidades nacionales diversas. Dado esto, ¿cómo concebir de la misma forma la aplicación de los estándares internacionales?

Estas dificultades pueden ser ilustradas en al menos tres maneras.

Primero, ¿cómo pensar en todos lados los mismos conceptos cuando las economías y los niveles de desarrollo son diferentes? ¿Se puede aplicar seriamente reglas idénticas en el Perú, por un lado, y en Europa y en Estados Unidos, por otro, cuando las problemáticas de desarrollo, sobre todo económicas, son distintas? Por comparación, en el Perú, la deuda pública es de aproximadamente $30 \%$ del PBI, y el crecimiento es más fuerte, pero también más desigual.

Además, los conceptos subyacentes de la buena gobernanza de las finanzas públicas, aquellos de transparencia, responsabilidad (accountability) o, incluso, de control no tienen las mismas acepciones alrededor del mundo, tanto en los vocabularios como en las tradiciones políticas y jurídicas.

Finalmente, cabe recordar las diferencias en cuanto a la visión del Estado, de las cuales se deriva el rol de la intervención pública que lo implementa, sobre todo en cuanto a la economía. Existen diversos modelos en lo que se refiere a este rol del Estado. Si se limita el análisis a los países denominados «occidentales», se tiene, por un lado, un modelo más bien liberal (Estados Unidos) que no cree que el interés general sobrepasa la suma de los intereses individuales; así, el Estado no necesita ocuparse de todo y debe respetar los intereses privados. Con este punto de partida, el gasto público y los impuestos son menores, pero los gastos privados son importantes (educación, salud, etc.). Por el contrario, otros países, sobre todo Francia, piensan que el Estado es el único que puede imponer el interés general. Los gastos públicos, y por tanto los impuestos, son, en este caso, consecuentes. Al hacer esto, dadas las desigualdades, las prescripciones que buscan supervisar al Estado, hacerlo responsable, son más fáciles de satisfacer en los países de tradición liberal. Si bien no existe un modelo que sea mejor que el otro, se trata, sobre todo, de tomar en cuenta la idea esencial de que existen modelos de Estado y de intervención pública, y, por lo tanto, modelos de finanzas públicas. Desde este punto de vista, ¿cómo entender la aplicación uniforme de estándares que, en el contexto actual, están más bien marcados por lógicas liberales más cercanas a los modelos anglosajones?

En resumen, el one size fits all es complejo, lo cual está demostrado por la aplicación de la regla de oro en legislaciones nacionales europeas que, sin embargo, tienen vínculos reales. 


\subsection{El ejemplo de la recepción diversa de la «regla de oro de las finanzas públicas» en Europa}

Parece juicioso enfocarse en la temática que es central en las recomendaciones y prácticas recientes: la del equilibrio de las cuentas públicas. La pregunta es la siguiente: ¿qué se sabe, en los hechos, de la recepción de la regla de oro en las legislaciones nacionales? ¿Es idéntica o al menos cercana?

Incluso si se piensa solamente en Europa continental —que agrupa países con vínculos culturales y jurídicos reales-, la respuesta es, por lo menos: presenta contrastes. Para probarlo, se debe empezar recordando que la Unión Europea, empujada por la crisis del 2008, reforzó la supervisión de las finanzas públicas de los Estados miembros, incitando fuertemente a que estos incluyan el equilibrio presupuestario en su derecho nacional (sobre todo a través del TSCG citado anteriormente). Algunos países - en primer lugar, Italia y España- modificaron sus constituciones en este sentido. En cuanto a Alemania, esta ya había incorporado en el 2009 en su texto supremo una disposición similar que limitaba los déficits tanto para el Gobierno federal como para los Länder. Por lo contrario, y así como otros países, Francia adoptó una solución muy diferente. Es cierto que modificó su Constitución para que sea conforme al TSCG, pero de manera específica, creando una nueva categoría de leyes: las leyes de programación de las finanzas públicas (plurianuales), que obligan a los presupuestos anuales a inscribirse dentro de ellas. Es cierto que estas últimas se integran dentro del «objetivo de equilibrio de las cuentas de las administraciones públicas» previsto por la Constitución francesa en su artículo 34; sin embargo, no existe un carácter vinculante, ya que el equilibrio es simplemente un «objetivo». En resumen, estamos frente a una obligación de medios y no de resultados.

De ello se desprende que la prescripción del equilibrio presupuestario, presentada hoy como una práctica central de la «buena gobernanza financiera», ni siquiera se aplica de forma análoga en países bastante cercanos, como los de Europa. Por lo tanto, es posible preguntarse: ¿cómo no sería también el caso con territorios mucho más diferentes alrededor del mundo?

De forma más general - y más allá de la pregunta sobre la inscripción de la regla de oro en las legislaciones nacionales-, la problemática también es la de su efectividad. Sin embargo, y contrariamente a Alemania, la introducción de esta regla en las constituciones de Italia y España no impidió que 
los Gobiernos de estos países quisieran adoptar presupuestos deficitarios ${ }^{6}$, lo cual generó reacciones reprobatorias de las instituciones europeas. ¿No es esto acaso una prueba adicional de lo difícil que es aplicar los mismos estándares, como también de la dificultad que tiene el derecho para organizar el ámbito financiero, que está en el corazón de la democracia parlamentaria?

En conclusión, si las finanzas públicas sanas son esenciales para un desarrollo económico y un crecimiento perennes, esto se logra con decisiones políticas que deben ser propias y adaptadas a cada país.

6 Utilizando derogaciones previstas por el derecho aplicable en ciertos casos «excepcionales» (recesión, etc.). 


\section{REFERENCIAS}

The World Bank. Sub-Saharan Africa: From crisis to sustainable growth, a long-term perspective study. Washington DC: The World Bank,1989.

Fonds Monétaire International (FMI). Code de bonnes pratiques en matière de transparence des finances publiques. FMI, 1998 (actualizado en el 2007).

«Transparence budgétaire: Les meilleures pratiques de l'OCDE». Revue de l'OCDE sur la gestion budgétaire $1, \mathrm{n} .^{\circ} 3(2001 / 2002)$.

\section{Sobre el autor}

Doctor en Derecho Público por la Universidad Panthéon-Assas, magíster por la Universidad de París II en Fiscalidad Empresarial y magíster summa cum laude en Derecho Comparado por la misma universidad. Profesor asociado de Derecho Público en la Universidad de París-Nanterre y miembro del Centro de investigación de Derecho Público (CRDP). Ha sido director de programas académicos de la Universidad de Nueva Caledonia y codirector de las Universidades de Verano Franco-Alemanas en Bielorrusia, Japón y Perú. 